\title{
How to Reduce Unnecessary Invasive Angiograms When Patients Are Initially Evaluated by Coronary Computed Tomography?
}

\author{
Edouard Cheneau ${ }^{1}$, Bruno Vahdat ${ }^{1}$, Noémie Resseguier ${ }^{2}$, Laurent Bernard ${ }^{1}$, Annamaria Molon ${ }^{1}$, \\ Roch Giorgi ${ }^{2}$, Dimitri Panagides ${ }^{1}$ \\ ${ }^{1}$ Service de Cardiologie, Clinique Bouchard, Marseille, France \\ ${ }^{2}$ Service de Santé Publique et d'Information Médicale, Hôpital de la Timone, Marseille, France \\ Email: edouard.cheneau@wanadoo.fr
}

Received November 5, 2012; revised December 16, 2012; accepted December 26, 2012

\begin{abstract}
Purpose: When Coronary Computed Tomography (CCT) detects coronary obstruction, patients are regularly referred to invasive angiogram. With higher sensitivity than specificity, CCT might induce unnecessary angiograms ("false positive CCT"). We sought to determine the patients and CCT findings associated with false positive CCT. Methods: Patients were prospectively referred to CCT with a 64-slice CT scan for suspected CAD. Inclusion criteria were: 1) clinically suspected angina pectoris; or 2) suspected silent ischemia on resting EKG. Exclusion criteria were acute coronary syndrome and non sinus rhythm. Invasive coronary angiogram (ICA) was performed on the basis of CCT findings (stenosis $>50 \%$ ). Analysis was performed on a per patient basis. Results: Out of 702 patients, 228 had suspected significant stenosis by CCT and ICA was performed in 176 patients. Coronary stenosis $>50 \%$ was not confirmed by ICA in 44 (25\%). In multivariate analysis, we observed that atypical angina (OR 3.63, CI 1.43 - 9.66), silent ischemia (OR 5.11, CI 1.89 - 14.6) and number of suspected stenosed arteries by CCT (OR 1.81, CI 1.15 - 2.94) were independently predictive of false positive CCT $(\mathrm{p}<0.05)$. Lesion location and coronary plaque characteristics did not impact on CCT accuracy. Conclusions: Performing CCT for atypical angina or silent ischemia is associated with higher rate of unnecessary invasive coronary angiograms. We failed to identify lesions characteristics prone to be "false positive" of CCT.
\end{abstract}

Keywords: Angina; Coronary Disease; Diagnosis; CCT

\section{Introduction}

Coronary computed tomography (CCT) is a rapidly developing technique and plays a rising role in angina diagnosis $[1,2]$. CCT has shown high sensibility for detecting obstructive coronary disease in a non-invasive manner $[3,4]$. Despite CCT can adequately rule out obstructive coronary artery disease (CAD), its high sensitivity is associated with a lower positive predictive value and a number of coronary stenosis on CCT are not confirmed on invasive coronary angiograms. Since CCT has a growing role as first line work-up for angina pectoris diagnosis, referring patients with a positive CCT to invasive coronary angiography might induce numerous unnecessary invasive angiograms [5]. From retrospective analysis, CCT usefulness appears to be dependent on pre-test probability for having CAD and might be most advantageous in subsets of patients, especially those with an intermediate risk [6]. Consequently, patient selection prior to CCT enables to increase CCT accuracy.

A complementary approach would be to individualize subset of patients or CCT findings that are more likely to be false positive of CCT after they were screened by this technique. In these patients, a positive CCT would not justify to directly undergo cardiac catheterization and alternative work-up might be developed. In this study, we sought to determine the patient characteristics and CCT findings associated with false positive CCT.

\section{Material and Method}

\subsection{Patients}

We included all patients referred to our center for CT scan as initial work up test to evaluate possible obstructive coronary heart disease. Patients presenting with typical or atypical angina pectoris or suspected silent ischemia on resting EKG were referred for CCT and prospectively included into the study. CCT is the frontline test routinely used in our institution for CAD diagnosis. Inclusion criteria were patients referred for CCT without previous myocardial stress imaging or treadmill 
test and patients with preceding equivocal stress test during a six month period.

A dedicated information sheet and database was used for data collection. Inclusions were opened from January 2009 to December 2010. Typical angina was defined as having three characteristics: 1) substernal discomfort; 2) that is precipitated by physical exertion or emotion; and 3) relieved with rest or sublingual nitroglycerin. Atypical angina pectoris was defined as having 2 of 3 of the definition characteristics. Suspected silent ischemia was defined as $\mathrm{Q}$ wave or negative $\mathrm{T}$ wave on resting $\mathrm{EKG}$ in at least in two derivations in patients with at least one risk factor. Inclusion criteria were patients referred for CCT as initial work up test and patients with preceding equivocal stress test. Exclusion criteria were non sinus rhythm, acute coronary syndromes, impaired renal function (serum creatinine $>120 \mu \mathrm{mol} / \mathrm{l}$ ) and known allergy to iodinated contrast material. The estimated pretest probability for obstructive CAD was estimated using the Duke Clinical Score, which includes type of chest discomfort, age, gender, and traditional risk factors [7]. The protocol was reviewed and accepted by the ethic committee of our Board of Cardiology.

\subsection{Scan Protocol}

Patients with a heart rate exceeding 70 beats/min received IV beta-blockers (Atenolol 5 to $15 \mathrm{mg}$ ) before CT examination. All scans were performed with a 64-slice CT scanner (Lightspeed VCT, General Electric Healthcare) that features a gantry rotation time of $350 \mathrm{~ms}$, a temporal resolution of $175 \mathrm{~ms}$, and a spatial resolution of $0.54 \mathrm{~mm}^{3}$. Tube voltage was $120 \mathrm{kV}$ ( $100 \mathrm{kV}$ for patients weight $<70 \mathrm{~kg}$ ), X-ray tube current was prospectively modulated on EKG (250 to $650 \mathrm{~mA}$ ). CT was acquired in a breath-hold and ECG-gated. Rotation speed and pitch were adjusted to the acquisition protocols and the heart rate. Calcium scoring was not performed. A bolus of contrast media (Ioxaglate $320 \mathrm{mG} \mathrm{I/mL}$, Guerbet) was infused in an antebrachial vein with use of a dual-barrel injector ( $70 \mathrm{cc}$ of contrast media at $5 \mathrm{cc} / \mathrm{s}$ washed out by $30 \mathrm{cc}$ of isotonic solution at $3.5 \mathrm{cc} / \mathrm{s}$ ). CT data were analyzed by the use of an offline Advantage Workstation (General Electric) using (curved) multiplanar reconstruction. Phases from 0 to $90 \%$ every $10 \%$ were systematically reconstructed to allow for imaging coronary arteries. Segments were scored positive for significant CAD if there was $\geq 50 \%$ diameter reduction of the lumen (at least $2 \mathrm{~mm}$ ) by visual assessment. Three experienced (more than 500 CT coronary angiograms) observers blinded about previous medical history and symptoms participated in the study. Optimal quality CT scan was defined as lumen visual assessment available in all $>2 \mathrm{~mm}$ diameter coronary arteries. Patients with non-optimal qual- ity CT (including heavy calcification without lumen assessment) were scored positive for significant CAD. All patients with significant stenosis by CCT were suggested to undergo to ICA.

\subsection{Angiographic Analysis}

Baseline quantitative angiography was performed using the contrast-filled injection catheter for image calibration. Cine Angiographic stenosis was defined as stenosis greater than 50\%. Quantitative coronary angiographic (QCA) analysis was performed using the Integris H5000C software (Philips, The Netherlands). Patients were classified as positive for significant CAD if at least one $(>2 \mathrm{~mm}$ diameter) coronary artery showed a significant stenosis $(>50 \%)$. Patients were classified as true positive CCT if they were classified as positive for CAD by both CCT and ICA and false positive CCT if significant stenosis by CCT was not confirmed by ICA.

\subsection{Statistics}

All statistical analyses were realized using the R software, version 2.12.0 (R Foundation for Statistical Computing, Vienna, Austria). First, a descriptive analysis of all recorded data was performed among the overall study population. Bivariate analysis was then performed by comparing the patients and lesions characteristics of the two groups, i.e. true positive CCT and false positive CCT. Continuous variables were expressed as mean \pm standard deviation, and were compared using t-test after graphically checking of the conditions of the test. Categorical variables were expressed as number of patients and percentages, and were compared using chi-square test or Fisher test, when appropriate. Variables were retained for the multivariate analysis when their effect had a p-value less than 0.25 for the bivariate analysis.

A multivariate logistic regression model was built. A backward stepwise selection procedure was applied to retain the significant $(\mathrm{p}<0.05)$ independent variables and their interactions in the final model. Significance was determined using likelihood ratio test. The accuracy of the final model was assessed by Hosmer-Lemeshow test (HLgof.test R package).

\section{Results}

We evaluated 702 patients (male 66\%) by CCT. Age was $61 \pm 11$ years. CCT image quality was inadequate in 74 patients $(10.5 \%)$. Significant obstruction was suspected in 228 patients $(32 \%)$ by CCT and invasive coronary angiogram was performed in 176 patients. Coronary stenosis was $>50 \%$ in 132 patients $(75 \%)$ by ICA. False positive CCT were observed in 44 patients $(25 \%)$.

Patients' characteristics were compared between true 
positive CCT and false positive CCT and Table 1 shows a higher rate of false positive CCT in women as compared to men, in atypical angina and silent ischemia as compared to typical angina symptoms. Lesion location and characteristics detected by CCT were also compared between the two groups (Table 2). We observed that false positive CCT was less likely when more coronary plaques and segment with obstruction were detected by CCT.

In multivariate analysis, atypical angina (OR 3.63, CI 1.43 - 9.66, $\mathrm{p}=0.008$ ), silent ischemia (OR 5.11, CI 1.89 $14.6, \mathrm{p}=0.002)$ and number of suspected stenosed arteries by CCT (OR 1.81, CI $1.15-2.94, \mathrm{p}=0.010)$ were predictive of false positive CCT (Table 3).

Table 1. Indicating patients characteristics.

\begin{tabular}{|c|c|c|c|}
\hline & $\begin{array}{c}\text { Stenosis by ICA } \\
\text { (True positive CCT) } \\
\mathrm{N}=132\end{array}$ & $\begin{array}{c}\text { No stenosis by ICA } \\
\text { (False positive CCT) } \\
\mathrm{N}=44\end{array}$ & $\mathrm{p}$ \\
\hline Age (years) & $64 \pm 12$ & $64 \pm 12$ & 0.96 \\
\hline Male & $105(80 \%)$ & $28(20 \%)$ & 0.03 \\
\hline Female & $27(64 \%)$ & $16(36 \%)$ & \\
\hline Previous CAD & $39(30 \%)$ & $7(16 \%)$ & 0.07 \\
\hline Pretest likelihood for CAD (\%) & $59 \pm 29$ & $53 \pm 32$ & 0.20 \\
\hline Angina typical & $69(52 \%)$ & $13(30 \%)$ & 0.03 \\
\hline Atypical & $37(28 \%)$ & $17(39 \%)$ & \\
\hline Silent ischemia & $26(20 \%)$ & $14(32 \%)$ & \\
\hline Hypercholesterolemia & $62(47 \%)$ & $26(59 \%)$ & 0.16 \\
\hline Diabetics & $35(26 \%)$ & $9(20 \%)$ & 0.42 \\
\hline Hypertension & $63(48 \%)$ & $19(43 \%)$ & 0.60 \\
\hline Smokers & $43(33 \%)$ & $10(23 \%)$ & 0.22 \\
\hline ST changes by rest EKG & $10(7.6 \%)$ & $3(6.8 \%)$ & $>0.99$ \\
\hline Q waves & $8(6.1 \%)$ & $0(0 \%)$ & 0.20 \\
\hline
\end{tabular}

Table 2. Indicating lesion characteristics observed by coronary computed tomography.

\begin{tabular}{|c|c|c|c|}
\hline & $\begin{array}{c}\text { Stenosis by ICA } \\
\text { (True positive CCT) } \\
\mathrm{N}=132\end{array}$ & $\begin{array}{c}\text { No stenosis by ICA } \\
\text { (False positive CCT) } \\
\mathrm{N}=44\end{array}$ & $\mathrm{p}$ \\
\hline Number of atherosclerotic plaques & $1.9 \pm 0.8$ & $1.6 \pm 0.9$ & 0.047 \\
\hline Number of plaques with stenosis $>50 \%$ & $1.5 \pm 1.0$ & $1.1 \pm 0.9$ & 0.036 \\
\hline Ostial lesions & $12(10 \%)$ & $8(22 \%)$ & 0.08 \\
\hline Calcified lesions & $93(73 \%)$ & $31(79 \%)$ & 0.43 \\
\hline Left Main & $6(5 \%)$ & $3(7 \%)$ & 0.69 \\
\hline LAD & $76(62 \%)$ & $19(53 \%)$ & 0.31 \\
\hline Diagonal & $14(11 \%)$ & $6(17 \%)$ & 0.40 \\
\hline LCX & $31(25 \%)$ & $4(11 \%)$ & 0.07 \\
\hline Obtuse marginal & $12(10 \%)$ & $2(6 \%)$ & 0.73 \\
\hline RCA & $50(41 \%)$ & $14(39 \%)$ & 0.82 \\
\hline Graft & $4(3 \%)$ & $0(0 \%)$ & 0.57 \\
\hline
\end{tabular}


Table 3. Indicating predictive factors for true positive corornary computed tomography by multivariate analysis.

\begin{tabular}{ccccc}
\hline & OR & Lower CI OR & Upper CI OR & \\
\hline Angina & & & & \\
Typical & 1 & & & \\
Atypical & 3.63 & 1.43 & 9.66 & 0.0076 \\
Silent & 5.11 & 1.89 & 0.0016 & 0.63 \\
Number of plaques with stenosis $>50 \%$ & 1.81 & 1.15 & 2.94 & 0.0130 \\
\hline
\end{tabular}

Explanations for false positive CCT were retrospectively analyzed in view of ICA (some patients with more than one explanation). They were related to stenosis overestimation $(\mathrm{N}=28)$, inadequate lumen measurement flanking calcified plaque $(\mathrm{N}=17)$ or ulcerated plaque $(\mathrm{N}$ $=3$ ), myocardial bridge $(\mathrm{N}=2)$, abnormal origin of the artery $(\mathrm{N}=1)$, and instent lumen assessment $(\mathrm{N}=4)$. Poor CCT imaging acquisition contributed to eleven cases of false positive CCT: supraventricular arrhythmia during acquisition $(\mathrm{N}=3)$, heart rate $>75 / \mathrm{min}(\mathrm{N}=2)$, breathing artifact $(\mathrm{N}=2)$.

Off note, 31 patients out of 144 patients correctly classified as significant CAD in the true positive CCT group had one lesion false positive by CCT.

\section{Discussion}

In this unselected group of patients with suspected CAD, a positive $\mathrm{CCT}$ followed by invasive coronary angiogram resulted in $25 \%$ of unnecessary invasive procedures. The lowest diagnostic yield of CCT was observed in patients with atypical angina and suspected silent ischemia. Secondary, we searched to establish whether some CCT findings were more likely to be false positive but we failed to individualize lesion patterns or localization in which CCT was more imprecise.

Risk stratification and diagnostic strategies are required for efficient utilization of ICA. However, the diagnostic yield of ICA remains low and recent studies suggest that one fourth to one half of patients who undergo ICA are found to have no obstructive CAD $[8,9]$. CCT has been compared with invasive coronary angiography for the detection of significant coronary artery lesions in several studies [10]. These studies demonstrated that significant coronary artery lesions can be identified with high sensitivity, especially since introduction of newer imaging modalities of 64 or more detectors tomography. Appropriate use criteria of CCT are growing and CCT is increasingly performed for CAD diagnosis [5]. When CCT detects coronary obstruction, patients are usually referred to invasive angiography to confirm coronary findings and establish treatment. Since CCT positive predictive value ranges from $80 \%$ to $95 \%$, a step by step approach with upstream CCT will likely be associated to unnecessary invasive angiograms [11,12]. In our study, we included unselected patients with suspected CAD and also observed that $25 \%$ of patients did not have obstructive CAD despite initial positive CCT finding. This result is encouraging and support findings that estimate a decrease in normal rate of ICA when cardiac CT programs are implemented [13]. CCT prevents to perform invasive angiography more frequently than it indicates the need for an 'unnecessary' invasive testing [14].

Since CCT is not a perfect "gatekeeper" to ICA, we investigated the patients characteristics associated to false positive CCT. We observed that patients with atypical angina and silent ischemia were not good candidate for CCT in this regard. These findings are also observed when functional testing is performed before ICA: a positive stress test has minimal effect on the predictive ability of diagnosis model of obstructive coronary artery disease [8]. Therefore, the diagnostic yield of CCT likely won't outperform functional tests in suspected silent ischemia.

Female gender is a consistent predictor of failed strategy for CAD diagnosis with functional tests and women have more frequently non obstructive invasive angiograms [9]. In our study, we did not show a lack of performance of CCT in women. Meijboom et al. found that positive predictive value of CCT was lower in women [15]. They attributed the difference to women having smaller arteries. Since CCT accuracy decreases with vessel size, higher rate of false positive findings are observed in women on a per-vessel analysis. In our study that included 240 women, we did not observe that women had a higher rate of normal angiogram in the multivariate analysis. Theoretical limitations of CCT in woman are not translated in large scale analysis on a perpatient basis. This encouraging finding suggests that a strategy with CCT as first line test might be useful to take over difficulties in evaluating chest pain in women [16]. Among patients characteristics, we also confirmed that age does not impact on CCT accuracy despite older age is associated with more calcified arteries [17]. This suggests that patients can undergo CCT imaging, independently 
from their age.

Once CCT detects coronary obstruction in epicardial coronary vessel, patients are commonly referred for catheterization. The goal is to confirm CCT findings and decision-making for potential revascularization. However, a greater focus should be placed on CCT findings and evaluate the likelihood of false positive CCT. That strategy might avoid unnecessary catheterization. In studies evaluating 64-slice CT to invasive coronary angiography, positive predictive value differed between vessels: it was lower in left circumflex and right coronary arteries as compared to left descending and left main arteries as well as in stent and distal segments as compared to native and proximal segments [18]. However, positive predictive value of CCT remained constantly high in a narrow range $(85 \%$ to $93 \%)$. As a result, lesion location impacted so slightly on CCT accuracy that anatomical features could not be predictive of false positive CCT. When we compared patients with or without confirmed coronary obstruction by ICA, we did not see difference in lesion characteristics or location. No anatomical subgroup can be identified as "prone to be false positive CCT". Though, we logically found that risk for false positive decreased when more plaques and more significant lesions were detected by CCT. Our per-patient analysis emphasizes the impact of the number of diseased vessels. Thus, patients with one vessel disease justify consideration before referring to catheterization although patients with more than one stenosed vessel by CCT can be more evidently directed to ICA. Performance of CCT was equivalent in calcified versus non-calcified plaques and lesion location did not impact on CCT accuracy. At a patient level, the CORE-64 trials did not find calcium score as a predictor of diagnostic accuracy of CCT [12]. Even if calcified lesions remain challenging in a per lesion analysis, this suggests that calcium does not decrease diagnosis accuracy on a per patient basis since the introduction of 64 detectors scans $[18,19]$.

Overall, we observed that image quality had the greatest impact on false positive CCT. Our data sustain the previously described influence of heart rate and rhythm on image reconstruction [19-21]. In addition, we have faced overestimation in stenosis measurement. This occurrence of stenosis overestimation is likely the most common mechanism leading to perform non obstructive invasive angiograms, especially in intermediate stenosis and complex lesions $[22,23]$. The definition of positive CCT we utilized was " $50 \%$ diameter reduction of the lumen by visual assessment" to determine significant stenosis by CCT. Even if this definition is commonly accepted, it might be insufficient to quantify CAD obstruction. New tools are developed to better evaluate lumen stenosis with automated quantification [24]. Thus, predictive value of CCT might increase with computed quantification, especially in high-quality image reconstruction [25]. Overall, we utilized best acquisition and reconstruction methods available at the time of the study inclusion. However, we didn't anticipate false positive CCT and did not implement our strategy in order to decrease those occurrences throughout the study. We cannot state that a higher lumen percent stenosis threshold might have impacted on "false positive" CCT rate.

This study had limited exclusion criteria. Therefore, it reflects a "real life" study evaluating coronary artery disease in a wide spectrum of patients. No functional testing was requested in the inclusion criteria and the impact of such test on CCT accuracy has not been evaluated. Secondly, the negative predictive value of CCT has not been established in this study. Definition for positive CCT might have been too restrictive and increase positive predictive value and lower negative predictive value of CCT. Nevertheless, we observed a negative predictive value of $99 \%$ in a previous study with same inclusion criteria, CCT methods and analysis criteria [26]. This suggests that definitions we utilized in the current study were adequately set in order to preserve high negative predictive value of CCT.

\section{Conclusion}

Performing CCT for atypical angina or silent ischemia is associated with higher rate of unnecessary invasive coronary angiograms. We failed to identify lesions characteristics prone to be "false positive" of CCT. Improving CCT imaging and lumen stenosis measurement appear the best means to reduce the rate of unnecessary angiogram following CCT.

\section{REFERENCES}

[1] M. Henneman, J. D. Schuijf, J. M. van Werkhoven, G. Pundziute, E. E. van der Wall, J. W. Jukema and J. J. Bax "Multi-Slice Computed Tomography Coronary Angiography for Ruling Out Suspected Coronary Artery Disease: What Is the Prevalence of a Normal Study in a General Clinical Population?" European Heart Journal, Vol. 29, No. 16, 2008, pp. 2006-2013. doi:10.1093/eurheartj/ehn284

[2] L. H. Piers, R. Dikkers, T. P. Willems, B. de Smet, M. Oudkerk, F. Zijlstra and R. A. Tio, "Computed Tomographic Angiography or Conventional Coronary Angiography in Therapeutic Decision-Making," European Heart Journal, Vol. 29, No. 23, 2008, pp. 2902-2907. doi:10.1093/eurheartj/ehn454

[3] W. B. Meijboom, M. F. Meijs, J. D. Schuijf, M. J. Cramer, N. R. Mollet, C. A. van Mieghem, K. Nieman, J. M. van Werkhoven, G. Pundziute, A. C. Weustink, A. M. de Vos, F. Pugliese, B. Rensing, a J. W. Jukem, J. J. Bax, M. Prokop, P. A. Doevendans, M. G. Hunink, G. P. Krestin and P. J. de Feyter, "Diagnostic Accuracy of 64-Slice Computed Tomography Coronary Angiography: A Prospec- 
tive, Multicenter, Multivendor Study," Journal of the American College of Cardiology, Vol. 52, No. 25, 2008, pp. 2135-2144. doi:10.1016/j.jacc.2008.08.058

[4] M. W. von Ballmoos, B. Haring, P. Juillerat and H. Alkadhi, "Meta-Analysis: Diagnostic Performance of LowRadiation-Dose Coronary Computed Tomography Angiography," Annals of International Medicine, Vol. 154, No. 6, 2011, pp. 413-420.

[5] A. J. Taylor, M. Cerqueira, J. M. Hodgson, D. Mark, J. Min, P. O'Gara and G. D. Rubin, "Appropriate Use Criteria for Cardiac Computed Tomography. A report of the American College of Cardiology Foundation Appropriate Use Criteria Task Force, the Society of Cardiovascular Computed Tomography, the American College of Radiology, the American Heart Association, the American Society of Echocardiography, the American Society of Nuclear Cardiology, the North American Society for Cardiovascular Imaging, the Society for Cardiovascular Angiography and Interventions, and the Society for Cardiovascular Magnetic Resonance," Circulation, Vol. 122, 2010, pp. e525-e555.

doi:10.1161/CIR.0b013e3181fcae66

[6] W. B. Meijboom, C. van Mieghem, N. R. Mollet, F. Pugliese, A. C. Weustink, N. van Pelt, F. Cademartiri, K. Nieman, E. Boersma, P. de Jaegere, G. P. Krestin and P. J. de Feyter, "64-Slice Computed Tomography Coronary Angiography in Patients with High, Intermediate, or Low Pretest Probability of Significant Coronary Artery Disease," Journal of the American College of Cardiology, Vol. 50, No. 15, 2007, pp. 1469-1475. doi:10.1016/j.jacc.2007.07.007

[7] D. B. Pryor, L. Shaw, C. B. McCants, K. L. Lee, D. B. Mark, F. E. Harrell Jr., L. H. Muhlbaier and R. M. Califf, "Value of the History and Physical in Identifying Patients at Increased Risk for Coronary Artery Disease," Annals of International Medicine, Vol. 118, No. 2, 1993, pp. 81-90.

[8] M. R. Patel, E. D. Peterson, D. Dai, J. M. Brennan, R. F. Redberg, H. V. Anderson, R. G. Brindis and P. S. Douglas, "Low Diagnostic Yield of Elective Coronary Angiography,"New England Journal of Medicine, Vol. 362, No. 5, 2010, pp. 886-895. doi:10.1056/NEJMoa0907272

[9] C. M. Taylor, K. H. Humphries, A. Pu, W. Ghali, M. Gao, M. Knudtson, U. Hoffmann, R. G. Carere, "A Proposed Clinical Model for Efficient Utilization of Invasive Coronary Angiography,"American Journal of Cardiology, Vol. 106, No. 4, 2010, pp. 457-462. doi:10.1016/j.amjcard.2010.03.061

[10] M. Hamon, R. Morello, J. W. Riddell and M. Hamon, "Coronary Arteries: Diagnostic Performance of 16-versus 64-Section Spiral CT Compared with Invasive Coronary Angiography-Meta-Analysis," Radiology, Vol. 245, No. 3, 2007, pp. 720-731. doi:10.1148/radiol.2453061899

[11] M. J. Budoff, D. Dowe, J. G. Jollis, M. Gitter, J. Sutherland, E. Halamert, M. Scherer, R. Bellinger, A. Martin, R. Benton, A. Delago, J. K. Min, "Diagnostic Performance of 64-Multidetector Row Coronary Computed Tomographic Angiography for Evaluation of Coronary Artery Stenosis in Individuals without Known Coronary Artery Disease: Results from the Prospective Multicenter Accuracy (Assessment by Coronary Computed Tomo- graphic Angiography of Individuals Undergoing Invasive Coronary Angiography) Trial,"Journal of the American College of Cardiology, Vol. 52, No. 21, 2008, pp. 17241732. doi:10.1016/i.jacc.2008.07.031

[12] J. M. Miller, C. E. Rochitte, M. Dewey, A. Arbab-Zadeh, H. Niinuma, I. Gottlieb, N. Paul, M. E. Clouse, E. P. Shapiro, J. Hoe, A. C. Lardo, D. E. Bush, A. de Roos, C. Cox, J. Brinker and J. A. Lima, "Diagnostic Performance of Coronary Angiography by 64-Row CT," New England Journal of Medicine, Vol. 359, No. 22, 2008, pp. 2324 2336. doi:10.1056/NEJMoa0806576

[13] B. J. Chow, A. Abraham, G. A. Wells, L. Chen, T. D. Ruddy, Y. Yam, N. Govas, P. D. Galbraith, C. Dennie and R. S. Beanlands, "Diagnostic Accuracy and Impact of Computed Tomographic Coronary Angiography on Utilization of Invasive Coronary Angiography," Circ Cardiovasc Imaging, Vol. 2, No. 1, 2009, pp. 16-23. doi:10.1161/CIRCIMAGING.108.792572

[14] J. Hausleiter, T. Meyer, M. Hadamitzky, M. Zankl, P. Gerein, K. Dörrler, A. Kastrati, S. Martinoff and A. Schömig, "Non-Invasive Coronary Computed Tomographic Angiography for Patients with Suspected Coronary Artery Disease: The Coronary Angiography by Computed Tomography with the Use of a Submillimeter Resolution (Cactus) Trial," European Heart Journal, Vol. 28, No. 24, 2007, pp. 3034-3041

[15] W. B. Meijboom, A. C. Weustink, F. Pugliese, C. A. van Mieghem, N. R. Mollet, N. van Pelt, F. Cademartiri, K. Nieman, E. Vourvouri, E. Regar, G. P. Krestin, P. J. de Feyter, "Comparison of Diagnostic Accuracy of 64-Slice Computed Tomography Coronary Angiography in Women versus Men with Angina Pectoris," American Journal of Cardiology, Vol. 100, No. 10, 2007, pp. 1532-1537. doi:10.1016/j.amjcard.2007.06.061

[16] J. H. Mieres, L. J. Shaw, A. Arai, M. J. Budoff, S. D. Flamm, W. G. Hundley, T. H. Marwick, L. Mosca, A. R. Patel, M. A. Quinones, R. F. Redberg, K. A. Taubert, A. J. Taylor, G. S. Thomas and N. K. Wenger, "Role of Noninvasive Testing in the Clinical Evaluation of Women with Suspected Coronary Artery Disease: Consensus Statement from the Cardiac Imaging Committee, Council on Clinical Cardiology, and the Cardiovascular Imaging and Intervention Committee, Council on Cardiovascular Radiology and Intervention, American Heart Association," Circulation, Vol. 111, No. 5, 2005, pp. 682-696. doi:10.1161/01.CIR.0000155233.67287.60

[17] M. Dewey, A. L. Vavere, A. Arbab-Zadeh, J. M. Miller, L. Sara, C. Cox, I. Gottlieb, K. Yoshioka, N. Paul, J. Hoe, A. de Roos, A. C. Lardo, J. A. Lima, M. E. Clouse, "Patient Characteristics as Predictors of Image Quality and Diagnostic Accuracy of MDCT Compared with Conventional Coronary Angiography for Detecting Coronary Artery Stenoses: CORE-64 Multicenter International Trial," American Journal of Roentgenology, Vol. 194, No. 1, 2010, pp. 93-102. doi:10.2214/AJR.09.2833

[18] P. D. Stein, A. Y. Yaekoub, F. Matta and H. D. Sostman, "64-Slice CT for Diagnosis of Coronary Artery Disease: A Systematic Review," American Journal of Medicine, Vol. 121, No. 8, 2008, pp. 715-725. doi:10.1016/j.amjmed.2008.02.039 
[19] G. L. Raff, M. J. Gallagher, W. W. O’Neill, J. A. Goldstein, "Diagnostic Accuracy of Noninvasive Coronary Angiography Using 64-Slice Spiral Computed Tomography," Journal of the American College of Cardiology, Vol. 46, No. 3, 2005, pp. 552-557. doi:10.1016/j.jacc.2005.05.056

[20] H. Brodoefel, C. Burgstahler, I. Tsiflikas, A. Reimann, S. Schroeder, C. D. Claussen, M. Heuschmid and A. F. Kopp, "Dual-Source CT: Effect of Heart Rate, Heart Rate Variability, and Calcification on Image Quality and Diagnostic Accuracy," Radiology, Vol. 247, No. 2, 2008, pp. 346-355. doi:10.1148/radiol.2472070906

[21] S. Leschka, S. Wildermuth, T. Boehm, L. Desbiolles, L. Husmann, A. Plass, P. Koepfli, T. Schepis, B. Marincek, P. A. Kaufmann and H. Alkadhi, "Noninvasive Coronary Angiography with 64-Section CT: Effect of Average Heart Rate and Heart Rate Variability on Image Quality," Radiology, Vol. 241, No. 2, 2006, pp. 378-385. doi:10.1148/radiol.2412051384

[22] H. Gouya, O. Varenne, L. Trinquart, E. Touzé, O. Vignaux, C. Spaulding, J. L. Mas and J. L. Sablayrolles Coronary Artery Stenosis in High-Risk Patients: 64-Section CT and Coronary Angiography_Prospective Study and Analysis of Discordance," Radiology, Vol. 252, No. 2, 2009, pp. 377-385. doi:10.1148/radiol.2522081271

[23] B. M. Wertman, V. Y. Cheng, S. Kar, H. Gransar, R. A.
Berg, H. Naik, R. Makkar, J. Friedman, J. Schapira and D. Berman, "Characterization of Complex Coronary Artery Stenosis Morphology by Coronary Computed Tomographic Angiography," JACC Cardiovasc Imaging, Vol. 2, No. 8, 2009, pp. 950-958. doi:10.1016/j.jcmg.2008.12.032

[24] S. Busch, T. R. Johnson, K. Nikolaou, F. von Ziegler, A. Knez, M. F. Reiser and C. R. Becker, "Visual and Automatic Grading of Coronary Artery Stenoses with 64-Slice CT Angiography in Reference to Invasive Angiography," European Radiology, Vol. 17, No. 6, 2007, pp. 14451451. doi:10.1007/s00330-006-0512-y

[25] M. J. Boogers, J. D. Schuijf, P. H. Kitslaar, J. M. van Werkhoven, F. R. de Graaf, E. Boersma, J. E. van Velzen, J. Dijkstra, I. M. Adame, L. J. Kroft, A. de Roos, J. H. Schreur, M. W. Heijenbrok, J. W. Jukema, J. H. Reiber and J. J. Bax, "Automated Quantification of Stenosis Severity on 64-Slice CT: A Comparison with Quantitative Coronary Angiography," JACC CardiovasC Imaging, Vol. 3, No. 7, 2010, pp. 699-709. doi:10.1016/j.jemg.2010.01.010

[26] E. Cheneau, B. Vahdat, L. Bernard, A. Molon, D. Panagides, "Routine Use of Coronary Computed Tomography as Initial Diagnostic Test for Angina Pectoris," Archives of Cardiovascular Diseases, Vol. 104, No. 1, 2011, pp. 29-34. doi:10.1016/j.acvd.2010.11.007 